\title{
Joint Beam-Hopping Scheduling and Power Allocation in NOMA-Assisted Satellite Systems
}

\author{
Anyue Wang ${ }^{1}$, Lei Lei ${ }^{1}$, Eva Lagunas ${ }^{1}$, Symeon Chatzinotas ${ }^{1}$, Ana Isabel Pérez Neira ${ }^{2}$, and Björn Ottersten ${ }^{1}$ \\ ${ }^{1}$ Interdisciplinary Centre for Security, Reliability and Trust (SnT), University of Luxembourg, Luxembourg \\ ${ }^{2}$ Centre Tecnològic de Telecomunicacions de Catalunya (CTTC/CERCA), Castelldefels, Spain \\ ${ }^{1,2}$ Emails: \{anyue.wang; lei.lei; eva.lagunas; symeon.chatzinotas; bjorn.ottersten $\} @$ uni.lu, ana.perez@cttc.es
}

\begin{abstract}
In this paper, we investigate potential synergies of non-orthogonal multiple access (NOMA) and beam hopping (BH) for multi-beam satellite systems. The coexistence of $\mathrm{BH}$ and NOMA provides time-power-domain flexibilities in mitigating a practical mismatch effect between offered capacity and requested traffic per beam. We formulate the joint $\mathrm{BH}$ scheduling and NOMA-based power allocation problem as mixed-integer nonconvex programming. We reveal the exponential-conic structure for the original problem, and reformulate the problem to the format of mixed-integer conic programming (MICP), where the optimum can be obtained by exponential-complexity algorithms. A greedy scheme is proposed to solve the problem on a timeslotby-timeslot basis with polynomial-time complexity. Numerical results show the effectiveness of the proposed efficient suboptimal algorithm in reducing the matching error by $62.57 \%$ in average over the OMA scheme and achieving a good trade-off between computational complexity and performance compared to the optimal solution.
\end{abstract}

Index Terms-NOMA, beam hopping, multi-beam satellite systems, resource allocation, mixed-integer conic programming.

\section{INTRODUCTION}

Satellite resources are scarce and expensive, necessitating the improvement of resource utilization to embrace the dramatic growth of broadband data traffic [1]. However, in conventional multi-beam satellite systems, on-board resources (e.g., power or bandwidth) are evenly distributed and all beams are permanently illuminated [2], often failing to match offered capacity to heterogeneous traffic distribution among beams. In this case, poor quality of service arises in beams with unmet traffic demand while resources are wasted in beams with excess capacity [1].

Beam hopping $(\mathrm{BH})$ is a promising technique to provide flexibility in resource management for satellite systems [3], [4]. Unlike conventional systems, only a subset of spot beams are illuminated at each timeslot in a $\mathrm{BH}$ system [1]. In this way, power consumption and the mass of satellite payload can be reduced for cost saving since fewer radio-frequency chains are required [5]. Beam illumination pattern, or beamtimeslot assignment, can be optimized based on traffic profile to reduce unmet and unused capacity [6], [7]. Besides, by illuminating beams that are distant from each other, beams adjacent to these illuminated beams are inactive, and thus cochannel interference can be largely alleviated [8]. Despite the abovementioned advantages, the spectral efficiency of conventional $\mathrm{BH}$ is inevitably limited by the existence of inactive beams [1], which invites the question of how to further improve spectral efficiency. The authors in [6] introduced precoding in $\mathrm{BH}$ systems to mitigate the co-channel interference resulting from illuminating a cluster of adjacent beams and, thus, further improve the performance of offered-requested rate matching. In [9], network coding was introduced to provide flexibility in the link layer to adapt to short-term traffic variation. The exploitation of more flexibility in resource management and further improvement in spectral efficiency for satellite $\mathrm{BH}$ systems is still an open issue [1].

Power-domain non-orthogonal multiple access (NOMA) is potentially advantageous to $\mathrm{BH}$ systems by providing an extra degree of freedom in the power domain [10]. With superposition coding and successive interference cancellation (SIC), NOMA is capable of multiplexing more than one terminal in the same frequency and time resource [11]. Compared to orthogonal multiple access (OMA) techniques, NOMA can obtain gains in spectral efficiency and accommodating more terminals [11], attracting researchers to investigate how NOMA can help multi-beam satellite systems [10]. The authors in [12] studied the synergy of NOMA and precoding to improve capacity in multi-beam satellite systems. In [13], NOMA was applied to allow the cooperation among adjacent beams and further accelerate the spectral efficiency. In [14], we investigated flexible resource allocation in a NOMA-enabled multibeam satellite system to improve the performance of offeredcapacity-to-requested-traffic ratio. However, it is still unclear how NOMA can cooperate with $\mathrm{BH}$ to further improve the spectral efficiency for satellite systems. To the best of our knowledge, this is the first work to investigate the potential synergies of NOMA and BH to provide time-power-domain flexibilities in overcoming the traffic-capacity mismatch issue.

In this paper, we consider a multi-beam satellite system where NOMA is implemented within the illuminated beams of a beam-hopped system in order to serve multiple terminals with the same spectrum resource. The metric of matching error [15], i.e., the square of the traffic-capacity gap, is adopted to capture the mismatch performance of each beam. The smaller the matching error is, the better match between traffic and capacity is achieved, and specifically, zero reflects a perfect 


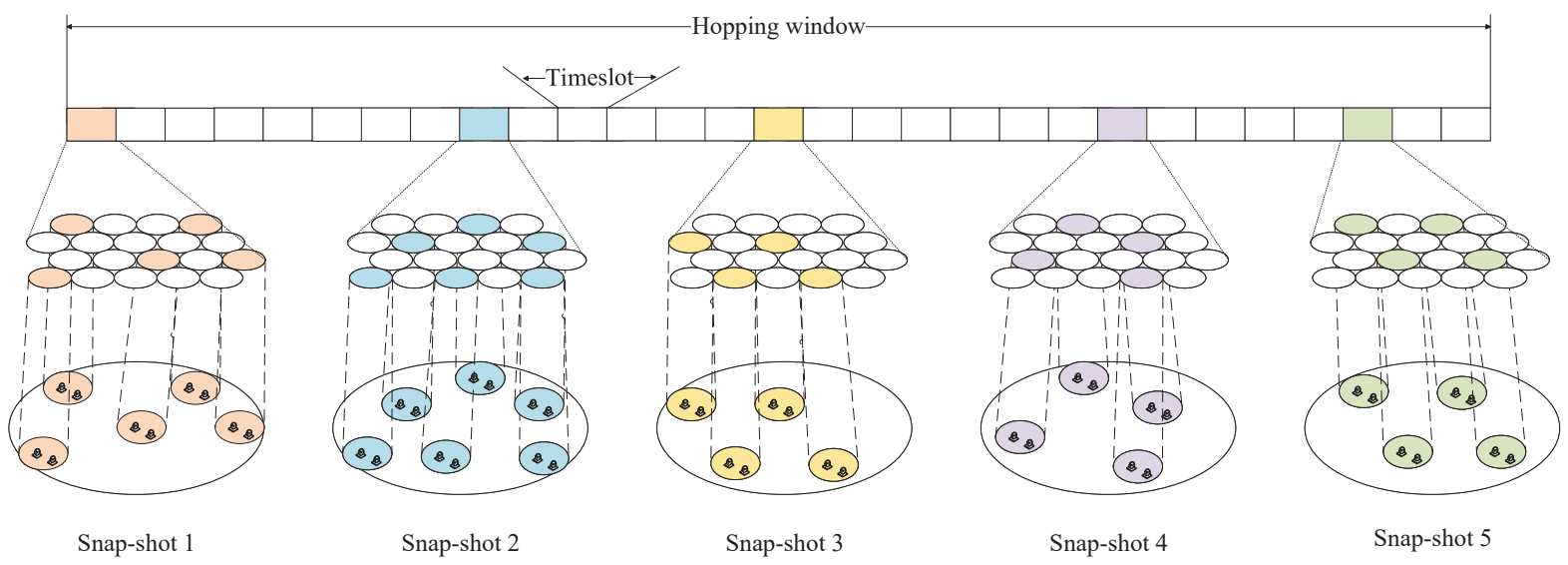

Fig. 1: An instance of one hopping window in a NOMA-assisted satellite BH system is illustrated. One snap-shot (of which the illuminated beams are highlighted in colors) is scheduled at each timeslot. Within each illuminated beam, NOMA is applied to multiplex two terminals. The figure presents beam illumination of 5 timeslots.

match. We formulate a problem to minimize the total matching error by jointly optimizing power allocation and beam illumination. The problem appears as a mixed-integer non-convex programming which is inherently complicated and with the variables' number exponentially growing as the number of beams increases. We first design a pre-processing to eliminate the combinations of illuminated beams that suffer from interbeam interference or do not meet payload constraints such that the number of variables can be reduced and the problem size can shrink. Then the problem is identified as a mixedinteger conic programming (MICP). The optimal or nearoptimal solution can be obtained by conventional algorithms but with exponential-time complexity. Thus we propose a suboptimal algorithm to provide solutions of power allocation and beam illumination timeslot by timeslot with polynomialtime complexity. Numerical results show the effectiveness of the proposed scheme compared to the benchmarks.

The remainders of this paper are organized as follows. The considered NOMA-assisted satellite BH system is illustrated in Section II. We formulate a joint problem of power allocation and beam illumination in Section III. A suboptimal solution on a timeslot-by-timeslot basis is designed in Section IV. In Section $\mathrm{V}$, we evaluate the performance of the proposed algorithm. Section VI concludes the paper.

\section{SySTEM MODEL}

We consider the forward-link transmission of a multi-beam satellite system with a total number of $B$ spot beams. In the service range of the $b$-th beam, there are $K_{b} \geq 1$ fixed ground terminals. Denote $\mathcal{B}$ and $\mathcal{K}_{b}$ as the sets of the beams, and the terminals in the $b$-th beam, respectively. Time granularity for resource allocation is set as one timeslot. Denote $\mathcal{T}$ as the set of the timeslots. The illustration of the considered system is presented in Fig. 1. In the system, NOMA is implemented within each selected active beam in order to multiplex more than one terminal with the same frequency band. Due to large propagation delay and flexible operation of beam illumination, the synchronization among gateways, satellite, and terminals is challenging [16]. For practical consideration, the pre-scheduled (pre-selected) BH scheme [5] is adopted. Beam illumination pattern is designed for long-term adaptation, e.g., from minutes to hours, and it is operated periodically such that terminals can remain stable in synchronization and receive signals at the right time [5], [16]. The periodic cycle is defined as one hopping window, consisting of $T$ timeslots. Assuming that terminals' channel gains have long coherence time and traffic demands remain invariant [15], we focus on resource optimization within one hopping window and replicate the allocation decision across hopping windows.

Based on the basis of $\mathrm{BH}$, one subset of $\mathcal{B}$ is selected at each timeslot and the beams in this subset are illuminated. Each subset is defined as one snap-shot. Denote $\mathcal{B}_{j}$ as the set of the beams in the $j$-th snap-shot and $\mathcal{J}$ as the set of the snap-shots, where $\mathcal{B}_{j} \subseteq \mathcal{B}$. The number of all the potential snap-shots is $J=2^{B}$, which exponentially grows as $B$ increases. Such exponentially growing property is an undesired issue for implementation. But the number of snap-shots can be reduced in practice. First, snap-shots illuminating adjacent beams since more inter-beam interference is introduced [7]. Second, practical limitations of satellite payload restrict beam illumination, e.g., a limited number of illuminated beams for cost-saving or energy-saving consideration [3]. Snap-shots that break these limitations can be eliminated.

Within each illuminated beam, NOMA is adopted to multiplex one or more terminals. According to the NOMA basis, signals of terminals in one beam are superimposed at the transmitter side. Each terminal receives the combination of the desired signals, intra-beam interference, inter-beam interference, and noise. The signal-to-interference-plus-noise ratio (SINR) of terminal $k$ in beam $b$ at timeslot $t$ when the beams in $\mathcal{B}_{j}$ are illuminated is derived as,

$$
\gamma_{b k t j}=\frac{\left|h_{b k}\right|^{2} \rho_{b k t j} P}{I_{b k t j}^{\text {intra }}+I_{b k t j}^{\text {inter }}+\sigma^{2}} .
$$

Here, $\left|h_{b k}\right|^{2}$ is the channel gain of terminal $k$ in beam $b . P$ is the maximum beam power. $\sigma^{2}$ is noise power. $\rho_{b k t j}, I_{b k t j}^{\mathrm{intra}}$, and $I_{b k t j}^{\text {inter }}$ are the power ratio, intra-beam interference, and inter-beam interference of terminal $k$ in beam $b$ at timeslot 
$t$ under snap-shot $j$, respectively. The inter-beam interference can be largely reduced and maintained at a low level when the illuminated beams are distant from each other. By eliminating snap-shots with adjacent illuminated beams, the inter-beam interference can be assumed negligible as $I_{b k t j}^{\text {inter }} \approx 0$. To mitigate intra-beam interference, SIC is adopted. For presentation simplification, we assume that the index of terminals is consistent with the descending order $\left|h_{b 1}\right|^{2}>\left|h_{b 2}\right|^{2}>$ $\cdots>\left|h_{b K_{b}}\right|^{2}$. For any two terminals $k_{1}$ and $k_{2}$, where $k_{1}<k_{2}, k_{1}$ decodes and removes $k_{2}$ 's signal whereas $k_{2}$ treats $k_{1}$ 's signal as noise. Intra-beam interference is expressed as $I_{b k t j}^{\text {intra }}=\sum_{k^{\prime}=1}^{k-1}\left|h_{b k}\right|^{2} \rho_{b k^{\prime} t j} P$ for $k>1$ and $I_{b k t j}^{\text {intra }}=0$ for $k=1$.

If snap-shot $j$ is scheduled, the rate of terminal $k$ in beam $b$ at timeslot $t$ is derived as

$$
R_{b k t j}=B_{W} \log \left(1+\gamma_{b k t j}\right),
$$

where $B_{W}$ is the occupied bandwidth. Denote $R_{b k}$ as the offered capacity of terminal $k$ in beam $b$. The offered capacity is expressed as $R_{b k}=\sum_{t \in \mathcal{T}} \sum_{j \in \mathcal{J}_{b}} R_{b k t j}$, where $\mathcal{J}_{b}=\left\{j^{\prime} \mid b \in \mathcal{B}_{j^{\prime}}, \forall j^{\prime} \in \mathcal{J}\right\}$ denotes the set of the snap-shots that includes beam $b$.

\section{PROBLEM FORMULATION}

In this section, we formulate an optimization problem to minimize the total error in matching offered capacity to requested traffic [15], i.e., $\left(R_{b}-D_{b}\right)^{2}$, by beam illumination and power allocation. Here, $R_{b}=\sum_{k \in \mathcal{K}_{b}} R_{b k}$ and $D_{b}$ are denoted as the aggregated capacity and demand of beam $b$, respectively. The variables are defined as,

$0 \leq \rho_{b k t j} \leq 1$, power ratio of terminal $k$ in beam $b$ at timeslot $t$ when the beams in $\mathcal{B}_{j}$ are illuminated.

$\alpha_{j t}=\left\{\begin{array}{l}1, \text { the beams in } \mathcal{B}_{j} \text { are illuminated at timeslot } t, \\ 0, \text { otherwise. }\end{array}\right.$

The problem is formulated as

$$
\begin{array}{ll}
\mathcal{P}_{1}: & \min _{\alpha_{j t}, \rho_{b k t j}} \sum_{b \in \mathcal{B}}\left(R_{b}-D_{b}\right)^{2} \\
\text { s.t. } & \sum_{j \in \mathcal{J}} \alpha_{j t}=1, \forall t \in \mathcal{T}, \\
& \sum_{k \in \mathcal{K}_{b}} \rho_{b k t j} \leq \alpha_{j t}, \forall b \in \mathcal{B}_{j}, t \in \mathcal{T}, j \in \mathcal{J}, \\
& R_{b k} \geq R_{b k}^{\min }, \forall b \in \mathcal{B}, k \in \mathcal{K}_{b}, \\
& \sum_{j \in \mathcal{J}_{b}} \sum_{t \in \mathcal{T}} \alpha_{j t} \geq 1, \forall b \in \mathcal{B} .
\end{array}
$$

Constraints (3b) state that only one snap-shot is scheduled at each timeslot. Constraints (3c) connect $\alpha$-variables and $\rho$ variables. If $\alpha_{j t}=1$, snap-shot $j$ is scheduled. Beam $b$ in $\mathcal{B}_{j}$ is illuminated at timeslot $t$ and the corresponding sum of the power ratio should be no larger than 1 . If $\alpha_{j t}=0$, $\sum_{k \in \mathcal{K}_{b}} \rho_{b k t j}$ is confined to 0 . Note that the constraints cannot restrict that $\sum_{k \in \mathcal{K}_{b}} \rho_{b k t j}>0, \forall b \in \mathcal{B}_{j}$, if $\alpha_{j t}=1$. This could result in the case that snap-shot $j$ is scheduled but there exists beam $b \in \mathcal{B}_{j}$ is allocated with zero power, which is equivalent to illuminating beams in $\mathcal{B}_{j^{\prime}}=\mathcal{B}_{j} \backslash\{b\}$. Thus these two cases can achieve the same objective at the optimum. Constraints (3d) restrict each terminal's offered capacity no smaller than the minimum rate limitation $R_{b k}^{\min }$. In (3e), each beam should be illuminated at least once during each hopping window. The reasons for introducing (3e) are two folds. On the one hand, terminals should be revisited considering terminals' capability of re-acquisition after a period of time without receiving messages [5]. On the other hand, terminals need to receive synchronization messages during each hopping window for stable communications [16].

Due to the presence of binary variables and the nonconvexity of the expression of $R_{b k}$ in (1) and (2), $\mathcal{P}_{1}$ appears mixed-integer non-convex programming [17], which is difficult to solve optimally. Before solving $\mathcal{P}_{1}$, two challenges need to be addressed. First, the numbers of binary and continuous variables are $J T$ and $\sum_{j \in \mathcal{J}} \sum_{b \in \mathcal{B}_{j}} K_{b} T$, respectively, which grow exponentially as $B$ increases. This issue can be tackled by removing the undesired snap-shots from $\mathcal{J}$, as mentioned in Section II. Specifically, we pre-process to omit snap-shots with the following properties:

- Illumination pattern does not meet the constraints of satellite payload, e.g., the number of illuminated beams surpasses the maximum limitation;

- Beams that are either adjacent or with inter-beam interference larger than a threshold are illuminated.

After this pre-processing, the number of variables is reduced so that the size of $\mathcal{P}_{1}$ can shrink to a certain extent.

Second, the convexity of $\mathcal{P}_{1}$ should be revealed. As interbeam interference is weak after the pre-processing, we assume it is negligible. We express $\rho_{b k t j}$ as the function of $R_{b k t j}$ based on (1) and (2) and derive the following equation [14],

$$
\sum_{k \in \mathcal{K}_{b}} \rho_{b k t j}=\sum_{k \in \mathcal{K}_{b}}\left(\frac{\sigma^{2}}{\left|h_{b k}\right|^{2}}-\frac{\sigma^{2}}{\left|h_{b(k-1)}\right|^{2}}\right) e^{\sum_{l \geq k} \frac{R_{b l t j}}{B_{W}}}-\frac{\sigma^{2}}{\left|h_{b K_{b}}\right|^{2}} \text {. }
$$

Then $\mathcal{P}_{1}$ is transformed equivalently into the following,

$$
\begin{aligned}
& \mathcal{P}_{2}: \min _{\alpha_{j t}, R_{b k t j}} \sum_{b \in \mathcal{B}}\left(R_{b}-D_{b}\right)^{2} \\
& \text { s.t. } \quad(3 \mathrm{~b}),(3 \mathrm{~d}),(3 \mathrm{e}), \\
& R_{b k t j} \leq R_{\max } \alpha_{j t}, \forall b \in \mathcal{B}_{j}, k \in \mathcal{K}_{b}, t \in \mathcal{T}, j \in \mathcal{J}, \\
& \sum_{k \in \mathcal{K}_{b}}\left(\frac{\sigma^{2}}{\left|h_{b k}\right|^{2}}-\frac{\sigma^{2}}{\left|h_{b(k-1)}\right|^{2}}\right) e^{\sum_{l \geq k} \frac{R_{b l t j}}{B_{W}}}-\frac{\sigma^{2}}{\left|h_{b K_{b}}\right|^{2}} \leq \alpha_{j t}, \\
& \qquad \forall b \in \mathcal{B}_{j}, t \in \mathcal{T}, j \in \mathcal{J},
\end{aligned}
$$

where $\alpha_{j t}$ and $R_{b k t j} \geq 0$ are variables. Note that $(5 \mathrm{c})$ is added to confine $R_{b k t j}=0$ if $\alpha_{j t}=0$, where $R_{\max }$ is no smaller than all $R$-variables. $\mathcal{P}_{2}$ is identified as mixed-integer convex programming. Specifically, it is a mixed-integer conic programming (MICP) [17] with quadratic cone in the objective and exponential cones in $(5 \mathrm{~d})$.

MICP can be obtained by conventional outer approximation or branch and bound approach, which can obtain the optimal or 
near-optimal solution. However, the computational complexity of the above solutions grows exponentially as the number of integer variables increases [18]. The inherent combinatorial and non-linear properties entail the intractability of MICP. For practical consideration, it is necessary to solve $\mathcal{P}_{2}$ with low computational complexity.

\section{Proposed Greedy Algorithm}

Considering the difficulties of attaining the optimum or near-optimum of MICP, we propose a suboptimal algorithm to solve $\mathcal{P}_{2}$ with polynomial-time complexity. The proposed algorithm is on the greedy basis to optimize power and beam illumination timeslot by timeslot. At the $t$-th timeslot, we solve the following subproblem,

$$
\begin{aligned}
& \mathcal{P}_{3}(t): \min _{\alpha_{j t}, R_{b k t j}} \sum_{b \in \mathcal{B}}\left(R_{b t}-D_{b}\right)^{2}+\sum_{b \in \mathcal{B}} \phi_{b}\left(\alpha_{j t}, R_{b k t j}\right) \\
& \text { s.t. } \sum_{j \in \mathcal{J}} \alpha_{j t}=1, \\
& R_{b k t j} \leq R_{\max } \alpha_{j t}, \forall b \in \mathcal{B}_{j}, k \in \mathcal{K}_{b}, j \in \mathcal{J}, \\
& \sum_{k \in \mathcal{K}_{b}}\left(\frac{\sigma^{2}}{\left|h_{b k}\right|^{2}}-\frac{\sigma^{2}}{\left|h_{b(k-1)}\right|^{2}}\right) e^{\sum_{l \geq k} \frac{R_{b l t j}}{B_{W}}}-\frac{\sigma^{2}}{\left|h_{b K_{b}}\right|^{2}} \leq \alpha_{j t}, \\
& \forall b \in \mathcal{B}, j \in \mathcal{J},
\end{aligned}
$$

where $R_{b t}=\sum_{\tau=1}^{t} \sum_{k \in \mathcal{K}_{b}} \sum_{j \in \mathcal{J}} R_{b k \tau j}$ denotes the accumulated offered capacity for beam $b$ from timeslot 1 to $t$. Besides, $\phi_{b}\left(\alpha_{j t}, R_{b k t j}\right)$ is introduced as a penalty function of beam $b$. The objective of $\mathcal{P}_{3}(t)$ is to minimize the sum of total matching error and the penalty at the $t$-th timeslot. The reason of introducing $\phi_{b}\left(\alpha_{j t}, R_{b k t j}\right)$ is that (3d) and (3e) are coupled among timeslots and thus cannot be simply decomposed into different timeslots. To guarantee the satisfaction of all the constraints at the end of the hopping window, we introduce $\phi_{b}\left(\alpha_{j t}, R_{b k t j}\right)$ to increase the penalties of the beams that do not meet (3d) and (3e) yet at the $t$-th timeslot. The penalty function is defined in the method of exact penalty [17] as,

$$
\begin{aligned}
\phi_{b}\left(\alpha_{j t}, R_{b k t j}\right)=\sum_{k \in \mathcal{K}} & \lambda_{b k} \max \left\{\left(R_{b k}^{\min }-\sum_{\tau=1}^{t} \sum_{j \in \mathcal{J}} R_{b k \tau j}\right), 0\right\} \\
& +\mu_{b} \max \left\{\left(1-\sum_{j \in \mathcal{J}_{b}} \sum_{\tau=1}^{t} \alpha_{j \tau}\right), 0\right\},
\end{aligned}
$$

where $\lambda_{b k}>0, \mu_{b}>0$ are penalty parameters for (3d) and (3e), respectively. The objective in $\mathcal{P}_{3}(t)$ is penalized if any terminals' capacity has not met the minimum rate constraint at timeslot $t$, and otherwise, the corresponding penalty is zero. Similarly, if the beam has not been illuminated, the related penalty is large. To minimize the objective, resources are prone to be assigned to the beams with large penalties.

$\mathcal{P}_{3}(t)$ is a MICP with $J$ binary and $\sum_{j \in \mathcal{J}} \sum_{b \in \mathcal{B}_{j}} K_{b}$ continuous variables and still seems complicated. The computational complexity needs to be further reduced. By setting $\alpha_{j t}=1$ and $\alpha_{j^{\prime} t}=0, \forall j^{\prime} \neq j$, we can decompose $\mathcal{P}_{3}(t)$ into $J$ snap-shots as,

$$
\mathcal{P}_{4}(t, j): \min _{R_{b k t j}} \sum_{b \in \mathcal{B}}\left(R_{b t}-D_{b}\right)^{2}+\sum_{b \in \mathcal{B}} \phi_{b}\left(\alpha_{j t}, R_{b k t j}\right)
$$

\section{$\overline{\text { Algorithm } 1 \text { Greedy scheme of beam illumination and power }}$} allocation

\section{Input:}

Penalty: $\lambda_{b k}, \mu_{b}$.

1: for $t=1, \ldots, T$ do

2: Obtain $y_{b t}$ by solving $\mathcal{P}_{5}(t, b), \forall b \in \mathcal{B}$.

3: $\quad$ Calculate $\bar{y}_{j t}=\sum_{b \in \mathcal{B}_{j}} y_{b t}$ for each snap-shot.

4: Schedule the snap-shot with the minimum objective value, i.e., $j^{*}=\arg \min _{j \in \mathcal{J}}\left\{\bar{y}_{j t}\right\}$, and set $\alpha_{j^{*} t}=1$.

5: end for

6: Obtain $R_{b k t j}$ and $\rho_{b k t j}$ by solving $\mathcal{P}_{1}$ with the determined beam illumination pattern.

\section{Output:}

$\alpha_{j t}, \rho_{b k t j}, R_{b k t j}$.

$$
\text { s.t. } \begin{aligned}
& \sum_{k \in \mathcal{K}_{b}}\left(\frac{\sigma^{2}}{\left|h_{b k}\right|^{2}}-\frac{\sigma^{2}}{\left|h_{b(k-1)}\right|^{2}}\right) e^{\sum_{l \geq k} \frac{R_{b l t j}}{B_{W}}}-\frac{\sigma^{2}}{\left|h_{b K_{b}}\right|^{2}} \\
& \leq 1, \forall b \in \mathcal{B},
\end{aligned}
$$

where the variables are $R_{b k t j} \geq 0$. Note that for all $j^{\prime} \neq j$, $R_{b k t j^{\prime}}=0 . \mathcal{P}_{4}(t, j)$ targets at obtaining the optimal power solution if the $j$-th snap-shot is scheduled. Define $\bar{y}_{j t}$ as the optimum of $\mathcal{P}_{4}(t, j)$. Then $\mathcal{P}_{3}(t)$ can be treated as the problem to select the optimal snap-shot with the minimum $\bar{y}_{j t}$, i.e., $j^{*}=\arg \min _{j \in \mathcal{J}}\left\{\bar{y}_{j t}\right\} . \mathcal{P}_{4}(t, j)$ can be further divided into $B$ beams as follow,

$$
\begin{aligned}
& \mathcal{P}_{5}(t, j, b): \min _{R_{b k t j}}\left(R_{b t}-D_{b t}\right)^{2}+\phi_{b}\left(\alpha_{j t}, R_{b k t j}\right) \\
& \text { s.t. } \quad \sum_{k \in \mathcal{K}_{b}}\left(\frac{\sigma^{2}}{\left|h_{b k}\right|^{2}}-\frac{\sigma^{2}}{\left|h_{b(k-1)}\right|^{2}}\right) e^{\sum_{l \geq k} \frac{R_{b l t j}}{B_{W}}}-\frac{\sigma^{2}}{\mid h_{\left.b K_{b}\right|^{2}}} \leq 1,
\end{aligned}
$$

where $R_{b k t^{\prime} j^{\prime}}=0$ for all $b^{\prime} \neq b$ and $j^{\prime} \neq j$. $\mathcal{P}_{5}(t, j, b)$ is to optimize power allocation when beam $b$ in snap-shot $j$ is illuminated at timeslot $t$. Since inter-beam interference is negligible as we eliminate the undesired snap-shots, power allocation within each illuminated beam is independent of other illuminated beams. Thus the optimal power allocation in beam $b$ is the same for $\forall j \in \mathcal{J}_{b}$. That means, for any two snap-shots, say $j_{1}$ and $j_{2}$ in $\mathcal{J}_{b}$, the optimum of $\mathcal{P}_{5}\left(t, j_{1}, b\right)$ is equivalent to that of $\mathcal{P}_{5}\left(t, j_{2}, b\right)$. We omit the index $j$ and rewrite $\mathcal{P}_{5}(t, j, b)$ as $\mathcal{P}_{5}(t, b)$. The problem is identified as conic programming. Denote the optimum of $\mathcal{P}_{5}(t, b)$ as $y_{b t}$. For each snap-shot, the optimum of $\mathcal{P}_{4}(t, j)$ can be derived as $\bar{y}_{j t}=\sum_{b \in \mathcal{B}_{j}} y_{b t}$.

The proposed greedy scheme is presented in Alg. 1. The algorithm behaves on a timeslot-by-timeslot basis. At each timeslot, $y_{b t}$, is obtained for each beam by solving $\mathcal{P}_{5}(t, b)$ in line 2. The optimum objective of each snap-shot, $\bar{y}_{j t}$, is derived in line 3 . In line 4, the snap-shot with the smallest $\bar{y}_{j t}$ is scheduled. After deciding all $\alpha$-variables, power and rate allocation is optimized by solving $\mathcal{P}_{2}$ with fixed binary variables. The number of iterations is $B T$ in total, where, in each iteration, a conic programming needs to be solved. For each conic programming, i.e., $\mathcal{P}_{5}(t, b)$, we adopt interior-point 
TABLE I: Simulation parameters

\begin{tabular}{c|c}
\hline Parameter & Value \\
\hline Frequency & $20 \mathrm{GHz}(\mathrm{Ka}$ band $)$ \\
Bandwidth, $B_{W}$ & $500 \mathrm{MHz}$ \\
Satellite location & $13^{\circ} \mathrm{E}$ \\
Satellite height & $35,786 \mathrm{~km}$ \\
Beam radiation pattern & Provided by ESA \\
Channel model & {$[7]$} \\
Number of beams, $B$ & 16 \\
in the context of [20] \\
Maximum transmit power per beam, $P$ & $20 \mathrm{dBW}$ \\
Noise power, $\sigma^{2}$ & $42.1 \mathrm{dBi}$ \\
Number of timeslots, $T$ & $-126.47 \mathrm{dBW}$ \\
Number of terminals per beam, $K_{b}$ & 256 \\
Maximum illuminated beams & 2 \\
\hline
\end{tabular}

TABLE II: The number of snap-shots versus $\nu$ after the preprocessing

\begin{tabular}{c|c|c|c|c|c|c}
\hline$\nu\left(* 10^{-14}\right)$ & 3.7 & 4.0 & 4.3 & 4.6 & 4.9 & 5.2 \\
\hline$J$ & 33 & 37 & 47 & 59 & 81 & 90 \\
\hline
\end{tabular}

method [19], which guarantees $\epsilon$-optimality with polynomialtime complexity $\mathcal{O}(-\eta \log (\epsilon))$ [19]. Here, $\epsilon>0$ denotes the maximum gap between the solved objective value and the optimum, and $\eta \geq 1$ denotes the parameter to construct the self-concordant barrier for the cones [19]. The total complexity of Alg. 1 is $\mathcal{O}(-B T \eta \log (\epsilon))$.

\section{Performance Evaluation}

In this section, we evaluate the performance of the proposed beam illumination and power allocation scheme in NOMAenabled satellite $\mathrm{BH}$ systems. The simulation parameters are summarized in TABLE I. The beam pattern is provided by European Space Agency (ESA) in the context of [20]. In each beam, 70 terminals are located. The simulation results are averaged over 1000 instances. For each instance, we randomly select $K_{b}$ terminals from each beam and serve them during the service period. The demand of each terminal is uniformly distributed. To testify the performance of the proposed scheme, we set the following schemes as benchmarks,

- NOMA opt: The optimal or near-optimal solution of $\mathcal{P}_{1}$ is obtained by MOSEK 9.0 [21], a powerful solver for MICP. NOMA-opt consumes large computational efforts but can be viewed as a lower bound to check the performance for the proposed scheme.

- OMA opt: The optimal solution is obtained when OMA is adopted [7]. In the OMA scheme, the frequency band is equally divided into $K_{b}$ subbands. Each subband is exclusively occupied by one terminal.

The number of all the possible snap-shots is $2^{16}$. By the pre-processing, we reduce the number to 2517 by omitting the snap-shots with more than 4 illuminated beams as defined in TABLE I. Then we eliminate the snap-shots with $\sum_{b \in \mathcal{B}_{j}}\left|h_{b^{\prime} b k}\right|^{2} \geq \nu$, where $h_{b^{\prime} b k}$ denotes the channel coefficient of terminal $k$ in beam $b$ when receiving the $b^{\prime}$-th beam's signal and $\nu$ denotes the threshold. TABLE II presents the number of snap-shots after the pre-processing, i.e., $J$, with respect to $\nu$.

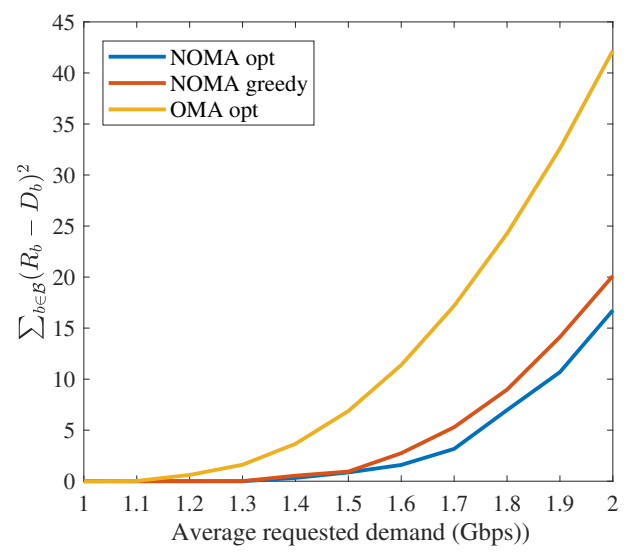

Fig. 2: Objective value versus average requested demand among NOMA opt, NOMA greedy scheme, and OMA opt.

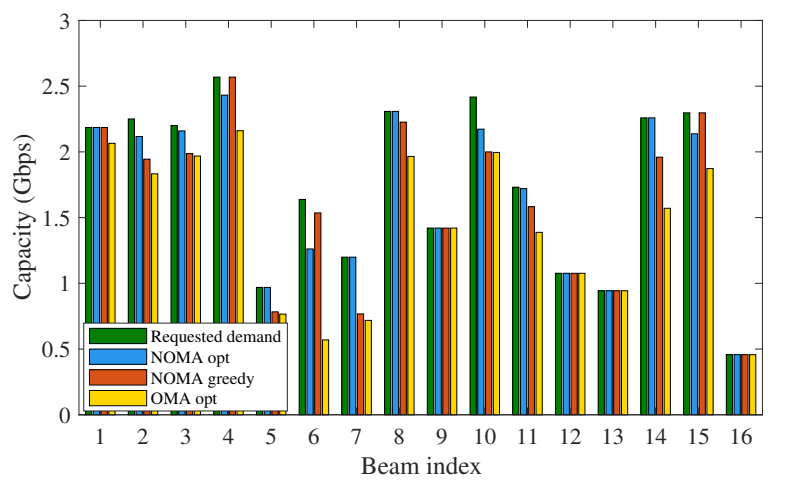

Fig. 3: An instance of the distribution of each beam's requested traffic demand and offered capacity assigned by three algorithms.

We first evaluate the performance of the objective value with respect to average requested demand among the three algorithms in Fig. 2. The number of snap-shots is 33. In general, the proposed greedy scheme can obtain about $62.57 \%$ performance gain in reducing the matching error compared to OMA opt scheme. When the average requested demand is relatively small (from $1 \mathrm{Gbps}$ to $1.5 \mathrm{Gbps}$ ), the proposed greedy scheme can achieve almost the same performance with NOMA opt. When the demand grows from 1.6 to $2 \mathrm{Gbps}$, the gap between NOMA greedy and NOMA opt rises from 1.15 to 3.37. For OMA opt, the matching error increases faster than both NOMA schemes. When the average demand reaches 2 Gbps, the gap between OMA opt and NOMA greedy scheme is 22.07 .

Next, Fig. 3 compares the distributions of each beam's requested demand and the offered capacity that is achieved by the three algorithms. NOMA opt can match offered capacity to requested demand better than the other two schemes, with an average traffic-capacity gap of $0.07 \mathrm{Gbps}$. We observe that NOMA greedy scheme performs worse in beams 2, 3, 5, 7, 11, and 14 than NOMA opt. But the loss is compensated as NOMA greedy scheme outperforms NOMA opt in beams 4, 6, and 15. The average gap between requested demand and offered capacity in NOMA greedy scheme is $0.137 \mathrm{Gbps}$. The reason for performance degradation is that NOMA greedy scheme 


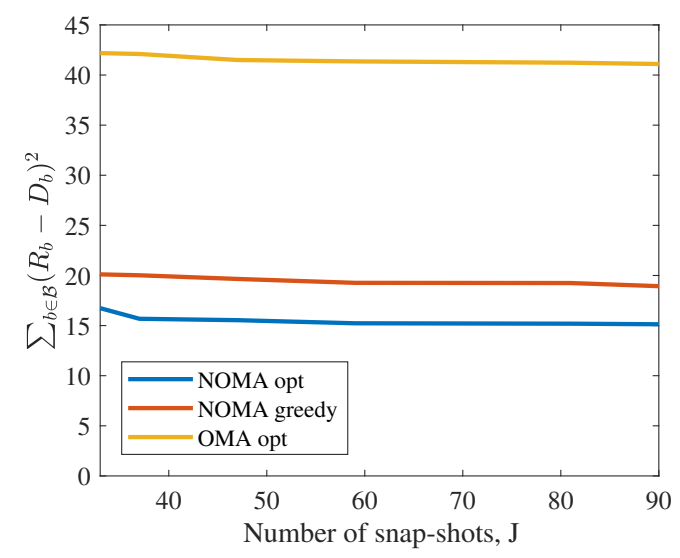

Fig. 4: Objective value versus the number of snap-shots, $J$. The average requested demand is $2 \mathrm{Gbps}$.

solves the problem of power allocation and beam illumination on a timeslot-by-timeslot basis, which achieves a local optimum with polynomial-time complexity while NOMA opt costs exponential computational time to attain the global optimum. OMA opt performs worse than both NOMA schemes with an average traffic-capacity gap of $3.33 \mathrm{Gbps}$. The proposed greedy scheme is able to achieve a good trade-off between the performance gain in requested-offered rate matching and computational efficiency.

At last, we verify that the pre-processing to eliminate the undesired snap-shots has trivial influence on the performance in Fig. 4. We observe that the objective values decrease slightly as the number of snap-shots increases from 33 to 90 by adjusting $\nu$ as shown in TABLE II. The percentages of the decrease in NOMA opt, NOMA greedy, and OMA opt are 9.62\%, 5.85\%, and $2.57 \%$, respectively. Among all $2^{16}$ possible snap-shots, only a small proportion (approximately $0.05 \%$ ) of snap-shots are desired to be scheduled to achieve a good performance. Meanwhile, the problem size and computational efforts are largely reduced. This result demonstrates the effectiveness of the pre-processing.

\section{CONCLUSION}

We have investigated the resource management in a NOMA$\mathrm{BH}$ coexisting system where $\mathrm{BH}$ is applied at the beam level while NOMA is implemented at the terminal level. The problem has been formulated to minimize the error in matching the requested-offered data rates by jointly allocating power and scheduling snap-shots. To solve the inherently complicated problem with low complexity, we have designed a pre-processing to reduce the size of the original problem and proposed a low-complexity suboptimal scheme to solve the problem timeslot by timeslot. Numerical results have shown the superiority of the proposed greedy scheme compared to benchmarks.

\section{ACKNOWLEDGMENT}

This work was supported in part by the European Research Council (ERC) project AGNOSTIC (under grant 742648), the FNR CORE project ROSETTA (under grant 11632107), the FNR CORE project FlexSAT (under grant 13696663).

\section{REFERENCES}

[1] O. Kodheli, E. Lagunas, N. Maturo, S. K. Sharma, B. Shankar, J. Montoya, J. Duncan, D. Spano, S. Chatzinotas, S. Kisseleff et al., "Satellite communications in the new space era: A survey and future challenges," arXiv preprint: 2002.08811, 2020.

[2] G. Maral, M. Bousquet, and Z. Sun, Satellite communications systems: systems, techniques and technology. John Wiley \& Sons, 2020.

[3] A. Freedman, D. Rainish, and Y. Gat, "Beam hopping how to make it possible," in Proc. Ka and Broadband Communication Conference, 2015.

[4] J. Anzalchi, A. Couchman, P. Gabellini, G. Gallinaro, L. D'agristina, N. Alagha, and P. Angeletti, "Beam hopping in multi-beam broadband satellite systems: System simulation and performance comparison with non-hopped systems," in 2010 5th Advanced Satellite Multimedia Systems Conference and the 11th Signal Processing for Space Communications Workshop. IEEE, 2010, pp. 248-255.

[5] A. Freedman, D. Rainish, and D. Elinav, "Beam hopping system design considerations," in 23rd Ka and Broadband Communications Conference.

[6] M. G. Kibria, E. Lagunas, N. Maturo, D. Spano, and S. Chatzinotas, "Precoded cluster hopping in multi-beam high throughput satellite systems," in 2019 IEEE Global Communications Conference (GLOBECOM). IEEE, 2019, pp. 1-6.

[7] L. Lei, E. Lagunas, Y. Yuan, M. G. Kibria, S. Chatzinotas, and B. Ottersten, "Beam illumination pattern design in satellite networks: Learning and optimization for efficient beam hopping," IEEE Access, vol. 8, pp. 136655-136667, 2020.

[8] J. Lei and M. A. Vazquez-Castro, "Multibeam satellite frequency/time duality study and capacity optimization," Journal of Communications and Networks, vol. 13, no. 5, pp. 472-480, 2011.

[9] R. Alegre-Godoy, N. Alagha, and M. A. Vázquez-Castro, "Offered capacity optimization mechanisms for multi-beam satellite systems," in 2012 IEEE International Conference on Communications (ICC). IEEE, 2012, pp. 3180-3184.

[10] A. I. Perez-Neira, M. Caus, and M. A. Vazquez, "Non-orthogonal transmission techniques for multibeam satellite systems," IEEE Communications Magazine, vol. 57, no. 12, pp. 58-63, 2019.

[11] S. R. Islam, N. Avazov, O. A. Dobre, and K.-S. Kwak, "Power-domain non-orthogonal multiple access (NOMA) in 5G systems: Potentials and challenges," IEEE Communications Surveys \& Tutorials, vol. 19, no. 2, pp. 721-742, 2016.

[12] M. Caus, M. Á. Vázquez, and A. Pérez-Neira, "NOMA and interference limited satellite scenarios," in 2016 50th Asilomar Conference on Signals, Systems and Computers. IEEE, 2016, pp. 497-501.

[13] N. A. K. Beigi and M. R. Soleymani, "Interference management using cooperative NOMA in multi-beam satellite systems," in 2018 IEEE International Conference on Communications (ICC). IEEE, 2018, pp. $1-6$.

[14] A. Wang, L. Lei, E. Lagunas, A. I. Perez-Neira, S. Chatzinotas, and B. Ottersten, "NOMA-enabled multi-beam satellite systems: Joint optimization to overcome offered-requested data mismatches," arXiv preprint: 2007.04718, 2020.

[15] G. Cocco, T. De Cola, M. Angelone, Z. Katona, and S. Erl, "Radio resource management optimization of flexible satellite payloads for DVBS2 systems," IEEE Transactions on Broadcasting, vol. 64, no. 2, pp. 266-280, 2017.

[16] X. Giraud, G. Lesthievent, and H. Méric, "Receiver synchronisation based on a single dummy frame for DVB-S2/S2X beam hopping systems," in 2018 25th International Conference on Telecommunications (ICT). IEEE, 2018, pp. 634-638.

[17] S. Boyd, S. P. Boyd, and L. Vandenberghe, Convex optimization. Cambridge university press, 2004.

[18] M. Köppe, "On the complexity of nonlinear mixed-integer optimization," in Mixed Integer Nonlinear Programming. Springer, 2012, pp. 533-557.

[19] S. P. Schurr, D. P. O'Leary, and A. L. Tits, "A polynomial-time interiorpoint method for conic optimization, with inexact barrier evaluations," SIAM Journal on Optimization, vol. 20, no. 1, pp. 548-571, 2009.

[20] ESA, "SATellite Network of EXperts (SATNEX) IV," https://satnex4. org/.

[21] M. ApS, "Mosek optimization toolbox for matlab," User's Guide and Reference Manual, Version, vol. 4, 2019. 